\title{
$M^{a} \vee$ e phenomena

\section{The nonlinear stochastic Schrödinger equation via stochastic Strichartz estimates}

Fabian Hornung

CRC Preprint 2016/32, November 2016

\section{KARLSRUHE INSTITUTE OF TECHNOLOGY}

\section{CRC 1173}

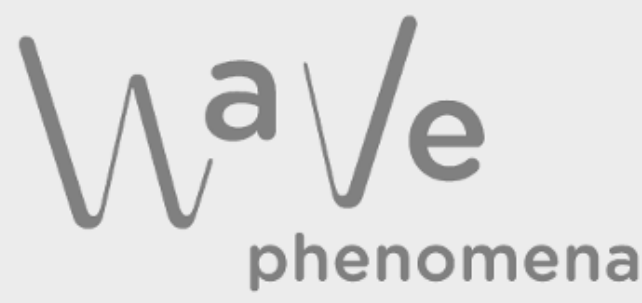




\section{Participating universities}

EBERHARD KARLS

UNIVERSITATT TUBINGEN

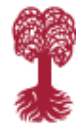

Funded by

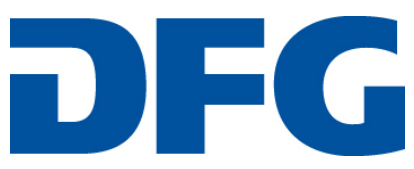

ISSN 2365-662X 


\title{
THE NONLINEAR STOCHASTIC SCHRÖDINGER EQUATION VIA STOCHASTIC STRICHARTZ ESTIMATES
}

\author{
FABIAN HORNUNG
}

\begin{abstract}
We consider the stochastic NLS with linear multiplicative noise in $L^{2}\left(\mathbb{R}^{d}\right)$ and prove the existence and uniqueness of a global solution in the subcritical and a local solution in the critical case, respectively. In particular, we relax the regularity assumptions on the noise from Barbu, Röckner and Zhang (Stochastic Nonlinear Schrödinger Equations with Linear Multiplicative Noise: Rescaling Approach. Journal of Nonlinear Science 24(3):383-409,2014). The proof is based on deterministic and stochastic Strichartz estimates.
\end{abstract}

Mathematics Subject Classification (2010): 35Q41, 35R60, 60H15, 60H30

Keywords: Nonlinear Schrödinger equation, Stratonovich Noise, Stochastic Strichartz estimates

\section{INTRODUCTION}

This article studies the following stochastic nonlinear Schrödinger equation

$$
\left\{\begin{aligned}
\mathrm{d} u(t) & =\left(\mathrm{i} \Delta u(t)-i \lambda|u(t)|^{\alpha-1} u(t)-\frac{1}{2} \sum_{m=1}^{\infty} B_{m}^{*} B_{m} u(t)\right) d t-\mathrm{i} \sum_{m=1}^{\infty} B_{m} u(t) \mathrm{d} \beta_{m}(t), \\
u(0) & =u_{0},
\end{aligned}\right.
$$

in $L^{2}\left(\mathbb{R}^{d}\right)$ with $\lambda \in\{-1,1\}, \alpha \in\left(1,1+\frac{4}{d}\right],\left(B_{m}\right)_{m \in \mathbb{N}} \subset \mathcal{L}\left(L^{2}\left(\mathbb{R}^{d}\right)\right)$ and independent Brownian motions $\left(\beta_{m}\right)_{m \in \mathbb{N}}$. The nonlinear Schrödinger equation can be seen as a model for nonlinear dispersive equation and enjoys physical significance in the description of nonlinear wave phenomena. In some situations, there is a random potential in the equation, which can be modeled by multiplicative Stratonovich noise. In [BCI $\left.{ }^{+94}\right]$, the equation (1.1) appears with parameters $d=2$ and $\alpha=3$, i.e. in the critical setting, in the context of Scheibe aggregates with thermal fluctuations.

In the literature, wellposedness of the NLS in $\mathbb{R}^{d}$ with multiplicative noise was studied by de Bouard and Debussche in [dBD99], [dBD03] followed by a series of papers concerning blow-up behavior and numerical studies (see [dBD02], [dBD ${ }^{+}$05], [DDM02], [DBD06]) and by Barbu, Röckner and Zhang in [BRZ14b],[BRZ16] and [BRZ14a]. In [BM13], Brzeźniak and Millet derived a new estimate for the stochastic convolution associated to the Schrödinger group. In contrast to [dBD99], where the authors work directly with the dispersive estimate of the Schrödinger group, the estimate from [BM13] is based on the deterministic Strichartz estimate. This allowed to prove global wellposedness for the NLS with Stratonovich noise on compact, two dimensional manifolds, where the dispersive estimate is not valid and has to be replaced by localized version, see [BGT04].

Date: November 22, 2016. 
In this article, we want to use the stochastic Strichartz estimate by Brzeźniak and Millet in order to solve the stochastic NLS in $L^{2}\left(\mathbb{R}^{d}\right)$ by a fixed point argument. We prove the following results for subcritical and critical nonlinearities, respectively.

Theorem 1.1. Let $u_{0} \in L^{2}\left(\mathbb{R}^{d}\right), \lambda \in\{-1,1\},\left(\beta_{m}\right)_{m \in \mathbb{N}}$ be a sequence of independent Brownian motions and $\left(B_{m}\right)_{m \in \mathbb{N}} \subset \mathcal{L}\left(L^{2}\left(\mathbb{R}^{d}\right)\right)$ with

$$
\sum_{m=1}^{\infty}\left\|B_{m}\right\|_{\mathcal{L}\left(L^{2}\left(\mathbb{R}^{d}\right)\right)}^{2}<\infty .
$$

Then, the following assertions hold:

a) Let $\alpha \in\left(1,1+\frac{4}{d}\right)$. Then, there is a unique global mild solution of $(1.1)$ in $L^{2}\left(\mathbb{R}^{d}\right)$.

b) Let $\alpha \in\left(1,1+\frac{4}{d}\right]$. Then, there is a unique local mild solution of $(1.1)$ in $L^{2}\left(\mathbb{R}^{d}\right)$.

We remark that the choice of the correction term is natural in the following sense. For selfadjoint operators $B_{m}$, we have

$$
\mathrm{i} B u(t) \circ \mathrm{d} W(t)=\mathrm{i} B u(t) \mathrm{d} W(t)-\frac{1}{2} \sum_{m=1}^{\infty} B_{m}^{2} u(t) \mathrm{d} t
$$

and therefore, (1.1) generalizes the stochastic NLS with Stratonovich noise.

We would like to compare our approach, assumptions and results to the articles [dBD99] by de Bouard and Debussche and [BRZ14b] by Barbu, Röckner and Zhang. For this purpose, we consider the special case of multiplication operators

$$
B_{m} u=e_{m} u, \quad u \in L^{2}\left(\mathbb{R}^{d}\right), \quad m \in \mathbb{N},
$$

with real valued functions $e_{m}, m \in \mathbb{N}$. The assumptions in [dBD99] correspond to the square function estimate

$$
\left\|\left(\sum_{m=1}^{\infty}\left|e_{m}\right|^{2}\right)^{\frac{1}{2}}\right\|_{L^{2}\left(\mathbb{R}^{d}\right) \cap L^{2+\delta}\left(\mathbb{R}^{d}\right)}<\infty
$$

for some $\delta>2(d-1)$ and they needed a restriction of the range of admissible exponents to $\alpha \in\left(1,1+\frac{2}{d-1}\right)$ for $d \geq 3$. As we see in Theorem 1.1. such a restriction can be avoided if we replace $(1.3)$ by

$$
\sum_{m=1}^{\infty}\left\|e_{m}\right\|_{L^{\infty}\left(\mathbb{R}^{d}\right)}^{2}<\infty
$$

To avoid estimates of the stochastic convolution needed in the fixed point arguments in the present paper and in [dBD99], Barbu, Röckner and Zhang use a different approach to problem (1.1) in [BRZ14b]. Via the scaling transformation $u=e^{-W} y$, they reduce (1.1) to a non-autonomous nonlinear Schrödinger equation with random coefficients, but without a stochastic integral.

Generally speaking, the main advantage of this approach is the fact that the equation can be solved pathwise, which allows to use known deterministic theory (see for example [Caz03], [LP14]) effectively. On the other hand, it is restricted to special situations, since the theory of Strichartz estimates for non-autonomous operators of the form

$$
A(s):=\mathrm{i}(\Delta+b(s) \cdot \nabla+c(s))
$$


is less developed than the theory for i $\Delta$. On $\mathbb{R}^{d}$, one can use results [Doi96] and [MMT08]. But a transfer of this method from $\mathbb{R}^{d}$ to a compact riemannian manifold $M$ to proof similar results as in [BM13] has not been done so far. Moreover, the deterministic Strichartz estimates for (1.5) need regular coefficients, which leads to the assumption

$$
\sum_{m=1}^{\infty}\left\|e_{m}\right\|_{C_{b}^{2}}^{2}<\infty
$$

and to the decay condition

$$
\lim _{|\xi| \rightarrow \infty} \eta(\xi)\left(\left|e_{m}(\xi)\right|+\left|\nabla e_{m}(\xi)\right|+\left|\Delta e_{m}(\xi)\right|\right)=0
$$

with

$$
\eta(\xi):= \begin{cases}1+|\xi|^{2}, & d \neq 2, \\ \left(1+|\xi|^{2}\right)\left(\log \left(2+|\xi|^{2}\right)\right)^{2}, & d=2 .\end{cases}
$$

Assuming (1.6) and (1.7) Barbu, Röckner and Zhang prove global wellposedness of (1.1) for $\alpha \in\left(1,1+\frac{4}{d}\right)$ and local wellposedness for $\alpha=1+\frac{4}{d}$, see [BRZ14b], Theorem 2.2 and Corollary 5.2. In view of (1.4), we are able to enlarge the class of admissible noises remarkably.

Let us briefly sketch the content of the present article. In the next section, we fix the notations and assumptions, introduce the solution concept and recall the deterministic and stochastic Strichartz estimates. In the third paragragh, we prove the local wellposedness of (1.1). To this end, we solve the problem

$$
\left\{\begin{aligned}
\mathrm{d} u_{n}(t) & =\left[\mathrm{i} \Delta u_{n}(t)-i \lambda F_{n}(u)(t)-\frac{1}{2} \sum_{m=1}^{\infty} B_{m}^{*} B_{m} u(t)\right] d t-\mathrm{i} \sum_{m=1}^{\infty} B_{m} u(t) \mathrm{d} \beta_{m}(t), \\
u(0) & =u_{0}
\end{aligned}\right.
$$

with the truncated nonlinearity $F_{n}(u)(t):=\theta_{n}\left(\|u\|_{L^{q}\left(0, t ; L^{\alpha+1}\right)}\right)|u(t)|^{\alpha-1} u(t)$, where $\theta_{n}:[0, \infty) \rightarrow$ $[0,1]$ is a cut-off-function, such that $\theta_{n}(x)=1$ for $x \in[0, n]$. This truncation permits the pathwise application of the deterministic Strichartz estimates in a fixed point argument in the natural space

$$
E_{[0, T]}:=L^{q}\left(\Omega, L^{q}\left(0, T ; L^{\alpha+1}\left(\mathbb{R}^{d}\right)\right) \cap C\left([0, T], L^{2}\left(\mathbb{R}^{d}\right)\right)\right),
$$

where $q$ is chosen such that $(\alpha+1, q)$ is a Strichartz pair. Since the solution of $(1.8)$ also solves (1.1) up to the stopping time

$$
\tau_{n}:=\inf \left\{t \geq 0:\left\|u_{n}\right\|_{L^{q}\left(0, t ; L^{\alpha+1}\right)} \geq n\right\} \wedge T
$$

this yields a local solution $u$ to (1.1) in the case $\alpha \in\left(1,1+\frac{4}{d}\right)$ up to time $\tau_{\infty}:=\sup _{n \in \mathbb{N}} \tau_{n}$. In the critical setting $\alpha=1+\frac{4}{d}$, an analogous argument yields a local solution. Note, that in this case, we use the truncation $\theta_{\nu}$ for a small $\nu \in(0,1)$ instead of $\theta_{n}$ for a large $n \in \mathbb{N}$.

The final section is concerned with the global existence of the solution in the subcritical case based on the uniform estimates

$$
\sup _{n \in \mathbb{N}} \mathbb{E}\left[\sup _{t \in[0, T]}\left\|u_{n}\right\|_{L^{2}}^{2}\right] \leq C_{T}, \quad \sup _{n \in \mathbb{N}} \mathbb{E}\left[\left\|u_{n}\right\|_{L^{q}\left(0, T ; L^{\alpha+1}\right)}\right] \leq C_{T} .
$$




\section{SETTING AND STRICHARTZ ESTIMATES}

In this section, we introduce some notations, assumptions and solution concepts and recall deterministic and stochastic Strichartz estimates, which will be used to construct the local solution.

Assumption 2.1. We assume the following:

i) We fix the space dimension $d \in \mathbb{N}$ and $T>0$. Moreover, let $u_{0} \in L^{2}\left(\mathbb{R}^{d}\right), \lambda \in\{-1,1\}$. We denote the Schrödinger group, i.e. the $C_{0}$-group of unitary operators generated by i $\Delta$, by $(U(t))_{t \in \mathbb{R}}$.

ii) Let $(\Omega, \mathcal{F}, \mathbb{P})$ be a probability space, $Y$ be a separable Hilbert space with $\mathrm{ONB}\left(f_{m}\right)_{m \in \mathbb{N}}$ and $W$ a cylindrical Wiener process in $Y$ adapted to a filtration $\mathbb{F}$ satisfying the usual conditions.

iii) Let $B: L^{2}\left(\mathbb{R}^{d}\right) \rightarrow \operatorname{HS}\left(Y, L^{2}\left(\mathbb{R}^{d}\right)\right)$ be a linear operator and set $B_{m} u:=B(u) f_{m}$ for $u \in L^{2}\left(\mathbb{R}^{d}\right)$ and $m \in \mathbb{N}$. Additionally, we assume that $B_{m}, m \in \mathbb{N}$, are bounded operators on $L^{2}\left(\mathbb{R}^{d}\right)$ with

$$
\sum_{m=1}^{\infty}\left\|B_{m}\right\|_{\mathcal{L}\left(L^{2}\right)}^{2}<\infty
$$

For presentation purposes, we used in the introduction that the process

$$
W=\sum_{m=1}^{\infty} f_{m} \beta_{m}
$$

with a sequence $\left(\beta_{m}\right)_{m \in \mathbb{N}}$ of independent Brownian motions is a cylindrical Wiener process in $Y$, see [DPZ14], Proposition 4.7. Since we look for mild solutions of (1.1), we reformulate the equation in the form

$$
u(t)=U(t) u_{0}+\int_{0}^{t} U(t-s)\left[-\mathrm{i} \lambda|u(s)|^{\alpha-1} u(s)+\mu(u(s))\right] \mathrm{d} s-\mathrm{i} \int_{0}^{t} U(t-s) B u(s) \mathrm{d} W(s) .
$$

In the following two Propositions, we introduce the main tool to apply a fixed argument to solve (2.2), namely the Strichartz estimates.

Proposition 2.2 (Deterministic Strichartz Estimates). Let $p_{j}, q_{j} \in[2, \infty], j=1,2$, with

$$
\frac{2}{q_{j}}+\frac{d}{p_{j}}=\frac{d}{2}, \quad\left(q_{j}, p_{j}, d\right) \neq(2, \infty, 2) .
$$

Let $x \in L^{2}\left(\mathbb{R}^{d}\right), J \subset \mathbb{R}$ an interval with $0 \in J$ and $f \in L^{q_{2}^{\prime}}\left(J, L^{p_{2}^{\prime}}\left(\mathbb{R}^{d}\right)\right)$. Then, there is a constant $C>0$ independent of $J, k, f$ and $x$ with

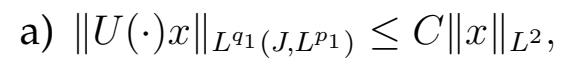

b) $\left\|\int_{0}^{\cdot} U(\cdot-s) f(s) \mathrm{d} s\right\|_{L^{q_{1}}\left(J, L^{p_{1}}\right)} \leq C\|f\|_{L^{q_{2}^{\prime}\left(J, L^{p_{2}^{\prime}}\right.}}$.

Furthermore, $U(\cdot) x$ and $\int_{0}^{\cdot} U(\cdot-s) f(s) \mathrm{d} s$ are elements of $C_{b}\left(J, L^{2}\left(\mathbb{R}^{d}\right)\right)$ and we have

c) $\|U(\cdot) x\|_{C_{b}\left(J, L^{2}\right)} \leq C\|x\|_{L^{2}}$,

d) $\left\|\int_{0}^{\cdot} U(\cdot-s) f(s) \mathrm{d} s\right\|_{C_{b}\left(J, L^{2}\right)} \leq C\|f\|_{L^{q_{2}^{\prime}\left(J, L^{p_{2}^{\prime}}\right.}}$.

Proof. These estimates are well known, see for example [Caz03], Theorem 2.3.3. 
The estimates from Proposition 2.2 can be used to deal with the free evolution and the deterministic convolution in (2.2). Furthermore, we need an estimate of the stochastic convolution. In order to apply Banach's fixed point Theorem iteratively, we have to deal with initial times $T_{0} \geq 0$. We denote the shifted filtration $\left(\mathcal{F}_{t+T_{0}}\right)_{t \geq 0}$ by $\mathbb{F}^{T_{0}}$. The process given by

$$
W^{T_{0}}(t):=W\left(T_{0}+t\right)-W\left(T_{0}\right), \quad t \geq 0,
$$

is a cylindrical Wiener process w.r.t. $\mathbb{F}^{T_{0}}$. For $T_{1}>0$ and a $\mathbb{F}^{T_{0}}$-predictable process $\Phi \in$ $L^{r}\left(\Omega, L^{2}\left(0, T_{1} ; \operatorname{HS}\left(Y, L^{2}\left(\mathbb{R}^{d}\right)\right)\right)\right)$, we define

$$
J_{\left[0, T_{1}\right]}^{T_{0}} \Phi(t):=\int_{0}^{t} U(t-s) \Phi(s) \mathrm{d} W^{T_{0}}(s), \quad t \in\left[0, T_{1}\right],
$$

by the stochastic integration theory in the Hilbert space $L^{2}\left(\mathbb{R}^{d}\right)$, see [DPZ14], chapter 4 . Note that for an $\mathbb{F}$-predictable process $\Phi$, we have

$$
\int_{0}^{t} U(t-s) \Phi\left(T_{0}+s\right) \mathrm{d} W^{T_{0}}(s)=\int_{T_{0}}^{T_{0}+t} U\left(\left(T_{0}+t\right)-s\right) \Phi(s) \mathrm{d} W(s)
$$

almost surely for all $t$. Since we are also interested in Strichartz estimates, we need a definition of the right hand side of $(2.3)$ in $L^{q}\left(0, T ; L^{p}\left(\mathbb{R}^{d}\right)\right)$-spaces for $q, p>2$. This can be done by the theory of stochastic integration in martingale type 2 spaces, see [Brz97] and the references therein or in UMD spaces, see [vNVW07]. The tool to estimate the stochastic convolution (2.3) is the following result due to Brzeźniak and Millet, [BM13].

Proposition 2.3 (Stochastic Strichartz Estimates). Let $T_{1}>0, r \in(1, \infty)$ and $p, q \in[2, \infty]$ with

$$
\frac{2}{q}+\frac{d}{p}=\frac{d}{2}, \quad(q, p, d) \neq(2, \infty, 2) .
$$

For all $\mathbb{F}^{T_{0}}$-predictable processes $\Phi \in L^{r}\left(\Omega, L^{2}\left(0, T_{1} ; \operatorname{HS}\left(Y, L^{2}\left(\mathbb{R}^{d}\right)\right)\right)\right), J_{\left[0, T_{1}\right]}^{T_{0}} \Phi$ is continuous in $L^{2}\left(\mathbb{R}^{d}\right)$ and $\mathbb{F}^{T_{0}}$-adapted with

$$
\left\|J_{\left[0, T_{1}\right]}^{T_{0}} \Phi\right\|_{L^{r}\left(\Omega, L^{q}\left(0, T_{1}, L^{p}\right)\right.} \lesssim\|\Phi\|_{L^{r}\left(\Omega, L^{2}\left(0, T_{1} ; \mathrm{HS}\left(Y, L^{2}\right)\right)\right)}
$$

and

$$
\left\|J_{\left[0, T_{1}\right]}^{T_{0}} \Phi\right\|_{L^{r}\left(\Omega, C\left(\left[0, T_{1}\right], L^{2}\right)\right.} \lesssim\|\Phi\|_{L^{r}\left(\Omega, L^{2}\left(0, T_{1} ; \mathrm{HS}\left(Y, L^{2}\right)\right)\right)}
$$

hold.

Proof. See [BM13], Theorem 3.10, Proposition 3.12. and Corollary 3.13 for the statement in the case $q=r$. The proof is based on the Burkholder-Gundy-Davis inequality in the martingale type 2 space $L^{q}\left(0, T_{1} ; L^{p}\left(\mathbb{R}^{d}\right)\right)$, see [Brz97], Theorem 2.4 , which holds for arbitrary $r \in(1, \infty)$. Therefore, $q=r$ is not needed.

Next, we introduce the Banach spaces for the fixed point argument depending on the power $\alpha$ of the nonlinearity. For $\alpha \in\left(1,1+\frac{4}{d}\right]$, we fix $q \in(2, \infty)$ such that

$$
\frac{2}{q}+\frac{d}{\alpha+1}=\frac{d}{2}
$$

in order to apply the Strichartz estimates from Propositions 2.2 and 2.3 with the exponents $(\alpha+1, q)$ and set

$$
Y_{[a, b]}:=L^{q}\left(a, b ; L^{\alpha+1}\left(\mathbb{R}^{d}\right)\right), \quad E_{[a, b]}:=Y_{[a, b]} \cap C\left([a, b], L^{2}\left(\mathbb{R}^{d}\right)\right), \quad 0<a<b .
$$


Furthermore, we abbreviate $Y_{r}:=Y_{[0, r]}$ and $E_{r}:=E_{[0, r]}$ for $r>0$. Let $\tau$ be an $\mathbb{F}$-stopping time. Then, we denote by $\mathbb{M}_{\mathbb{F}}^{q}\left(\Omega, E_{[0, \tau]}\right)$ the Banach space of continuous $\mathbb{F}$-adapted processes $u:[0, T] \times \Omega \rightarrow L^{2}\left(\mathbb{R}^{d}\right)$ with

$$
\|u\|_{\mathbb{M}_{\mathbb{R}}^{q}\left(\Omega, E_{[0, \tau]}\right)}^{q}:=\mathbb{E}\left[\sup _{t \in[0, \tau]}\|u(t)\|_{L^{2}}^{q}+\int_{0}^{\tau}\|u(t)\|_{L^{\alpha+1}}^{q} \mathrm{~d} t\right]<\infty .
$$

Often, we abbreviate $u \in \mathbb{M}_{\mathbb{F}}^{q}\left(\Omega, E_{\tau}\right):=\mathbb{M}_{\mathbb{F}}^{q}\left(\Omega, E_{[0, \tau]}\right)$. Moreover, we say $u \in \mathbb{M}_{\mathbb{F}}^{q}\left(\Omega, E_{[0, \tau)}\right)$ if $u$ is a continuous $\mathbb{F}$-adapted process in $L^{2}\left(\mathbb{R}^{d}\right)$ and there is a sequence $\left(\tau_{n}\right)_{n \in \mathbb{N}}$ of stopping times with $\tau_{n} \nearrow \tau$ almost surely as $n \rightarrow \infty$, such that $u \in \mathbb{M}_{\mathbb{F}}^{q}\left(\Omega, E_{\left[0, \tau_{n}\right]}\right)$ for all $n \in \mathbb{N}$.

Definition 2.4. Let $\alpha \in\left(1,1+\frac{4}{d}\right]$.

a) A local mild solution of (1.1) is a triple $\left(u,\left(\tau_{n}\right)_{n \in \mathbb{N}}, \tau\right)$ consisting of

- a stopping time $\tau$ and a sequence of stopping times $\left(\tau_{n}\right)_{n \in \mathbb{N}}$ with $\tau_{n} \nearrow \tau$ almost surely as $n \rightarrow \infty$,

- a process $u \in \mathbb{M}_{\mathbb{F}}^{q}\left(\Omega, E_{[0, \tau)}\right)$,

such that the equation

$$
\begin{gathered}
u(t)=U(t) u_{0}+\int_{0}^{t} U(t-s)\left[-\mathrm{i} \lambda|u(s)|^{\alpha-1} u(s)+\mu(u(s))\right] \mathrm{d} s \\
-\mathrm{i} \int_{0}^{t} U(t-s) B u(s) \mathrm{d} W(s)
\end{gathered}
$$

holds almost surely on $\left\{t \leq \tau_{n}\right\}$ in $L^{2}\left(\mathbb{R}^{d}\right)$ for all $n \in \mathbb{N}$. Often, we shortly denote the local mild solution by $(u, \tau)$.

b) Solutions of (1.1) are called unique, if we have

$$
\mathbb{P}\left(u_{1}(t)=u_{2}(t) \quad \forall t \in\left[0, \sigma_{1} \wedge \sigma_{2}\right)\right)=1
$$

for all local mild solutions $\left(u_{1}, \sigma_{1}\right)$ and $\left(u_{2}, \sigma_{2}\right)$.

c) A local mild solution $(u, \tau)$ with $\tau=T$ almost surely and $u \in \mathbb{M}_{\mathbb{F}}^{q}\left(\Omega, E_{[0, T]}\right)$ is called global mild solution.

\section{TRUNCATED EQUATION AND LOCAL WELLPOSEDNESS}

This section is devoted to the proof of the local part of Theorem 1.1. In order to transfer the deterministic fixed point argument, see [LP14], Theorems 5.2 and 5.3, to the stochastic setting, we would like to use the Strichartz estimates for the nonlinear term pathwise. On the other hand, Proposition 2.3 only gives us an $L^{r}(\Omega)$-estimate for the stochastic term at hand. To overcome this difficulty, we truncate the nonlinearity by a cutoff function $\theta_{n}$ and look for a mild solution of the equation

$$
\left\{\begin{aligned}
\mathrm{d} u_{n}(t) & =\left(\mathrm{i} \Delta u_{n}(t)-\mathrm{i} \theta_{n}\left(\left\|u_{n}\right\|_{L^{q}\left(0, t ; L^{\alpha+1}\right)}\right)\left|u_{n}(t)\right|^{\alpha-1} u_{n}(t)-\mu(u(t))\right) d t-\mathrm{i} B u_{n}(t) \mathrm{d} W(t), \\
u(0) & =u_{0} .
\end{aligned}\right.
$$

with fixed $n \in \mathbb{N}$. Here, $\theta_{n}:[0, \infty) \rightarrow[0,1]$ is defined by $\theta_{n}:=\theta\left(\frac{\dot{\bar{n}}}{n}\right)$ for

$$
\theta(x)= \begin{cases}1, & x \in[0,1], \\ 2-x, & x \in[1,2], \\ 0, & x \in[2, \infty) .\end{cases}
$$


In particular, we have $\theta_{n}(x)=1$ for $x \in[0, n]$ and

$$
\left|\theta_{n}(x)-\theta_{n}(y)\right| \leq \frac{1}{n}|x-y|, \quad x, y \geq 0 .
$$

To simplify the presentation, we use the following abbreviations for $r>0$ and $t \in[0, r]$ :

$$
\begin{gathered}
K_{\text {det }}^{n} u(t):=-\mathrm{i} \lambda \int_{0}^{t} U(t-s)\left[\theta_{n}\left(\|u\|_{Y_{s}}\right)|u(s)|^{\alpha-1} u(s)\right] \mathrm{d} s, \\
K_{\text {Strat }} u(t):=\int_{0}^{t} U(t-s) \mu(u(s)) \mathrm{d} s, \\
K_{\text {stoch }} u:=-\mathrm{i} J_{[0, r]}^{0} B u:=-\mathrm{i} \int_{0}^{t} U(t-s) B u(s) \mathrm{d} W(s) .
\end{gathered}
$$

Next, we introduce our notion of a solution of (3.1).

Definition 3.1. Let $\alpha \in\left(1,1+\frac{4}{d}\right]$.

a) A local mild solution of (3.1) is a pair $\left(u^{n}, \tau^{n}\right)$ consisting of a stopping time $\tau^{n} \in[0, T]$ and a process $u^{n} \in \mathbb{M}_{\mathbb{F}}^{q}\left(\Omega, E_{\tau^{n}}\right)$, such that the equation

$$
u^{n}=U(\cdot) u_{0}+K_{\text {det }}^{n} u^{n}+K_{\text {Strat }} u^{n}+K_{\text {stoch }} u^{n}
$$

holds almost surely on $\left\{t \leq \tau^{n}\right\}$ in $L^{2}\left(\mathbb{R}^{d}\right)$.

b) Solutions of (3.1) are called unique, if we have

$$
\mathbb{P}\left(u_{1}^{n}(t)=u_{2}^{n}(t) \quad \forall t \in\left[0, \sigma^{n} \wedge \tau^{n}\right)\right)=1
$$

for all local mild solutions $\left(u_{1}^{n}, \sigma^{n}\right)$ and $\left(u_{2}^{n}, \tau^{n}\right)$.

c) A local mild solution $\left(u^{n}, \tau^{n}\right)$ with $\tau^{n}=T$ almost surely is called global mild solution.

In the following Proposition, we state existence and uniqueness for (3.1).

Proposition 3.2. Let $\alpha \in\left(1,1+\frac{4}{d}\right)$. Then, there is a unique global mild solution $\left(u^{n}, T\right)$ of (3.1).

Proof. We fix $n \in \mathbb{N}$ and construct the solution from the assertion inductively.

Step 1: We look for a fixed point of the operator given by

$$
K^{n} u:=U(\cdot) u_{0}+K_{\text {det }}^{n} u+K_{\text {Strat }} u+K_{\text {stoch }} u, \quad u \in \mathbb{M}_{\mathbb{F}}^{q}\left(\Omega, E_{r}\right),
$$

where $r>0$ will be chosen small enough. Let $u \in \mathbb{M}_{\mathbb{F}}^{q}\left(\Omega, E_{r}\right)$. A pathwise application of Proposition 2.2 and integration over $\Omega$ yields

$$
\begin{gathered}
\left\|U(\cdot) u_{0}\right\|_{\mathbb{M}_{\mathbb{F}}^{q}\left(\Omega, E_{r}\right)} \lesssim\left\|u_{0}\right\|_{L^{2}} ; \\
\left\|K_{S t r a t} u\right\|_{\mathbb{M}_{\mathbb{F}}^{q}\left(\Omega, E_{r}\right)} \lesssim\|\mu(u)\|_{L^{q}\left(\Omega, L^{1}\left(0, r ; L^{2}\right)\right)}=\frac{1}{2}\left\|\sum_{m=1}^{\infty} B_{m}^{*} B_{m} u\right\|_{L^{q}\left(\Omega, L^{1}\left(0, r ; L^{2}\right)\right)} \\
\leq \frac{1}{2} \sum_{m=1}^{\infty}\left\|B_{m}\right\|_{\mathcal{L}\left(L^{2}\right)}^{2}\|u\|_{L^{q}\left(\Omega, L^{1}\left(0, r ; L^{2}\right)\right)} \lesssim\|u\|_{L^{q}\left(\Omega, L^{1}\left(0, r ; L^{2}\right)\right)} \leq r\|u\|_{\mathbb{M}_{\mathbb{F}}^{q}\left(\Omega, E_{r}\right)} .
\end{gathered}
$$

We define a stopping time by

$$
\tau:=\inf \left\{t \geq 0:\|u\|_{Y_{t}} \geq 2 n\right\} \wedge r
$$


and estimate

$$
\begin{aligned}
\left\|K_{d e t}^{n} u\right\|_{E_{r}} & \lesssim\left\|\theta_{n}\left(\|u\|_{Y .}\right)|u|^{\alpha-1} u\right\|_{L^{q^{\prime}}\left(0, r ; L^{\frac{\alpha+1}{\alpha}}\right)} \leq\left\||u|^{\alpha-1} u\right\|_{\left.L^{q^{\prime}\left(0, \tau ; L^{\frac{\alpha+1}{\alpha}}\right.}\right)} \\
& \leq\|u\|_{L^{q}\left(0, \tau ; L^{\alpha+1}\right)}^{\alpha} \tau^{\delta} \leq(2 n)^{\alpha} r^{\delta}
\end{aligned}
$$

using Proposition $2.2 b)$ and $d)$ and the Hölder inequality with $\delta:=1-\frac{d}{4}(1-\alpha)>0$. Integrating over $\Omega$ yields

$$
\left\|K_{\text {det }}^{n} u\right\|_{\mathbb{M}_{\mathbb{F}}^{q}\left(\Omega, E_{r}\right)} \lesssim(2 n)^{\alpha} r^{\delta}
$$

By Proposition 2.3, we obtain

$$
\begin{aligned}
\left\|K_{\text {stoch }} u\right\|_{\mathbb{M}_{\mathbb{F}}^{q}\left(\Omega, E_{r}\right)} & \lesssim\|B u\|_{L^{q}\left(\Omega, L^{2}\left(0, r ; \operatorname{HS}\left(Y, L^{2}\right)\right)\right)}=\left(\mathbb{E}\left(\int_{0}^{r} \sum_{m=1}^{\infty}\left\|B_{m} u\right\|_{L^{2}}^{2} \mathrm{~d} t\right)^{\frac{q}{2}}\right)^{\frac{1}{q}} \\
& \leq\left(\sum_{m=1}^{\infty}\left\|B_{m}\right\|_{\mathcal{L}_{\left(L^{2}\right)}}^{2}\right)^{\frac{1}{2}}\|u\|_{L^{q}\left(\Omega, L^{2}\left(0, r ; L^{2}\right)\right)} \lesssim r^{\frac{1}{2}}\|u\|_{L^{q}\left(\Omega, L^{\infty}\left(0, r ; L^{2}\right)\right)} \\
& \leq r^{\frac{1}{2}}\|u\|_{\mathbb{M}_{\mathbb{F}}^{q}\left(\Omega, E_{r}\right)} .
\end{aligned}
$$

Putting the estimates together, we get

$$
\left\|K^{n} u\right\|_{\mathbb{M}_{\mathbb{F}}^{q}\left(\Omega, E_{r}\right)} \lesssim\left\|u_{0}\right\|_{L^{2}\left(\mathbb{R}^{d}\right)}+(2 n)^{\alpha} r^{\delta}+\left(r+r^{\frac{1}{2}}\right)\|u\|_{\mathbb{M}_{\mathbb{F}}^{q}\left(\Omega, E_{r}\right)}
$$

for $u \in \mathbb{M}_{\mathbb{F}}^{q}\left(\Omega, E_{r}\right)$ and therefore the invariance of $\mathbb{M}_{\mathbb{F}}^{q}\left(\Omega, E_{r}\right)$ under $K^{n}$. To show the contractivity of $K^{n}$, we take $u_{1}, u_{2} \in \mathbb{M}_{\mathbb{F}}^{q}\left(\Omega, E_{r}\right)$ and get

$$
K^{n} u_{1}-K^{n} u_{2}=K_{\text {det }}^{n}\left(u_{1}\right)-K_{\text {det }}^{n}\left(u_{2}\right)+K_{\text {Strat }}\left(u_{1}-u_{2}\right)+K_{\text {stoch }}\left(u_{1}-u_{2}\right) .
$$

By the linearity of $K_{\text {Strat }}$ and $K_{\text {stoch }}$, the estimates

$$
\left\|K_{\text {Strat }}\left(u_{1}-u_{2}\right)\right\|_{\mathbb{M}_{\mathbb{F}}^{q}\left(\Omega, E_{r}\right)} \lesssim r\left\|u_{1}-u_{2}\right\|_{\mathbb{M}_{\mathbb{F}}^{q}\left(\Omega, E_{r}\right)}
$$

and

$$
\left\|K_{\text {stoch }}\left(u_{1}-u_{2}\right)\right\|_{\mathbb{M}_{\mathbb{F}}^{q}\left(\Omega, E_{r}\right)} \lesssim r^{\frac{1}{2}}\left\|u_{1}-u_{2}\right\|_{\mathbb{M}_{\mathbb{F}}^{q}\left(\Omega, E_{r}\right)}
$$

can be deduced as above. We define stopping times $\tau_{1}$ and $\tau_{2}$ by

$$
\tau_{j}:=\inf \left\{t \geq 0:\left\|u_{j}\right\|_{Y_{t}} \geq 2 n\right\} \wedge r, \quad j=1,2,
$$

and fix $\omega \in \Omega$. Without loss of generality, we assume $\tau_{1}(\omega) \leq \tau_{2}(\omega)$. We use the deterministic Strichartz estimates from Proposition 2.2

$$
\begin{aligned}
\left\|K_{\text {det }}^{n}\left(u_{1}\right)-K_{\text {det }}^{n}\left(u_{2}\right)\right\|_{E_{r}} \lesssim & \left.\left\|\theta_{n}\left(\left\|u_{1}\right\|_{Y .}\right)\left|u_{1}\right|^{\alpha-1} u_{1}-\theta_{n}\left(\left\|u_{2}\right\|_{Y .}\right)\left|u_{2}\right|^{\alpha-1} u_{2}\right\|_{L^{q^{\prime}(0, r ; L}} \frac{\alpha+1}{\alpha}\right) \\
\leq & \left\|\theta_{n}\left(\left\|u_{1}\right\|_{Y .}\right)\left(\left|u_{1}\right|^{\alpha-1} u_{1}-\left|u_{2}\right|^{\alpha-1} u_{2}\right)\right\|_{\left.L^{q^{\prime}\left(0, r ; L^{\frac{\alpha+1}{\alpha}}\right.}\right)} \\
& +\left\|\left[\theta_{n}\left(\left\|u_{1}\right\|_{Y .}\right)-\theta_{n}\left(\left\|u_{2}\right\|_{Y .}\right)\right]\left|u_{2}\right|^{\alpha-1} u_{2}\right\|_{\left.L^{q^{\prime}\left(0, r ; L^{\frac{\alpha+1}{\alpha}}\right.}\right)}
\end{aligned}
$$

By the properties of $\theta_{n}$ and the local Lipschitz estimate of the nonlinearity, we derive

$$
\begin{aligned}
\| \theta_{n}\left(\left\|u_{1}\right\|_{Y .}\right) & \left.\left(\left|u_{1}\right|^{\alpha-1} u_{1}-\left|u_{2}\right|^{\alpha-1} u_{2}\right) \|_{L^{q^{\prime}}(0, r ; L} \frac{\alpha+1}{\alpha}\right) \\
& \leq \tau_{1}^{\delta}\left(\left\|u_{1}\right\|_{L^{q}\left(0, \tau_{1}, L^{\alpha+1}\right)}+\|\left. u_{1}\right|^{\alpha-1} u_{1}-\mid u_{L^{q}\left(0, \tau_{1}, L^{\alpha+1}\right)}\right)^{\alpha-1}\left\|u_{1}-u_{2}\right\|_{L^{q}\left(0, \tau_{1}, L^{\alpha+1}\right)} \|_{L^{q^{\prime}}\left(0, \tau_{1} ; L^{\frac{\alpha+1}{\alpha}}\right)} \\
& \leq r^{\delta}(4 n)^{\alpha-1}\left\|u_{1}-u_{2}\right\|_{L^{q}\left(0, \tau_{1}, L^{\alpha+1}\right)} \leq r^{\delta}(4 n)^{\alpha-1}\left\|u_{1}-u_{2}\right\|_{E_{r}}
\end{aligned}
$$



and

$$
\begin{aligned}
\|\left[\theta_{n}\left(\left\|u_{1}\right\|_{Y .}\right)\right. & \left.-\theta_{n}\left(\left\|u_{2}\right\|_{Y .}\right)\right]\left|u_{2}\right|^{\alpha-1} u_{2}\left\|_{\left.L^{q^{\prime}\left(0, r ; L^{\frac{\alpha+1}{\alpha}}\right.}\right)} \leq \frac{1}{n}\right\|\left\|u_{1}-u_{2}\right\|_{Y .}\left|u_{2}\right|^{\alpha-1} u_{2} \|_{\left.L^{q^{\prime}\left(0, \tau_{2} ; L^{\frac{\alpha+1}{\alpha}}\right.}\right)} \\
& \leq \frac{1}{n}\left\|u_{1}-u_{2}\right\|_{Y_{r}}\left\|\left|u_{2}\right|^{\alpha-1} u_{2}\right\|_{\left.L^{q^{\prime}\left(0, \tau_{2} ; L^{\frac{\alpha+1}{\alpha}}\right.}\right)} \\
& \leq \frac{1}{n}\left\|u_{1}-u_{2}\right\|_{E_{r}} \tau_{2}^{\delta}\left\|u_{2}\right\|_{L^{q}\left(0, \tau_{2} ; L^{\alpha+1}\right)}^{\alpha} \leq \frac{1}{n}\left\|u_{1}-u_{2}\right\|_{E_{r}} r^{\delta}(2 n)^{\alpha} .
\end{aligned}
$$

We obtain

$$
\left\|K_{\text {det }}^{n}\left(u_{1}\right)-K_{\text {det }}^{n}\left(u_{2}\right)\right\|_{E_{r}} \lesssim\left(2^{\alpha}+4^{\alpha-1}\right) r^{\delta} n^{\alpha-1}\left\|u_{1}-u_{2}\right\|_{E_{r}} .
$$

Integrating over $\Omega$ and collection of the estimates for the other terms leads to

$$
\left\|K^{n}\left(u_{1}\right)-K^{n}\left(u_{2}\right)\right\|_{\mathbb{M}_{\mathbb{F}}^{q}\left(\Omega, E_{r}\right)} \lesssim\left[\left(2^{\alpha}+4^{\alpha-1}\right) r^{\delta} n^{\alpha-1}+r^{\frac{1}{2}}+r\right]\left\|u_{1}-u_{2}\right\|_{\mathbb{M}_{\mathbb{F}}^{q}\left(\Omega, E_{r}\right)} .
$$

Hence, there is a small time $r=r(n, \alpha)>0$, such that $K^{n}$ is a strict contraction in $\mathbb{M}_{\mathbb{F}}^{q}\left(\Omega, E_{r}\right)$ and Banach's Fixed Point Theorem yields $u^{n, 1} \in \mathbb{M}_{\mathbb{F}}^{q}\left(\Omega, E_{r}\right)$ with $K^{n}\left(u_{1}^{n}\right)=u_{1}^{n}$.

Step 2: We choose $r>0$ as in the first step and assume that we have $k \in \mathbb{N}$ and $u_{k}^{n} \in$ $\mathbb{M}_{\mathbb{F}}^{q}\left(\Omega, E_{k r}\right)$ with

$$
u_{k}^{n}=U(\cdot) u_{0}+K_{\text {det }}^{n} u_{k}^{n}+K_{\text {Strat }} u_{k}^{n}+K_{\text {stoch }} u_{k}^{n}
$$

on the interval $[0, k r]$. We want to extend $u_{k}^{n}$ to $[k r,(k+1) r]$. Therefore, we define the operators

$$
\begin{gathered}
K_{\text {det }, k}^{n} u(t):=-\mathrm{i} \lambda \int_{0}^{t} U(t-s)\left[\theta_{n}\left(\left(\left\|u_{k}^{n}\right\|_{Y_{k r}}^{q}+\|u\|_{Y_{s}}^{q}\right)^{\frac{1}{q}}\right)|u(s)|^{\alpha-1} u(s)\right] \mathrm{d} s, \\
K_{\text {stoch }, k} u(t):=-\mathrm{i} J_{[0, r]}^{k r} B u(t):=-\mathrm{i} \int_{0}^{t} U(t-s) B u(s) \mathrm{d} W^{k r}(s)
\end{gathered}
$$

for $t \in[0, r]$ and $u \in \mathbb{M}_{\mathbb{F} k r}^{q}\left(\Omega, E_{r}\right)$ and

$$
K_{k}^{n} u:=U(\cdot) u_{k}^{n}(k r)+K_{d e t, k}^{n} u+K_{\text {Strat }} u+K_{\text {stoch }, k} u, \quad u \in \mathbb{M}_{\mathbb{F}^{k r}}^{q}\left(\Omega, E_{r}\right),
$$

and search for a fixed point $v_{k+1}^{n}$ of $K_{k}^{n}$. The estimates for $K_{S t r a t}$ and $K_{\text {stoch, } k}$ are identical to the initial step. To estimate the deterministic convolution, we take $v_{1}, v_{2} \in \mathbb{M}_{\mathbb{F} k r}^{q}\left(\Omega, E_{r}\right)$ and define the stopping times

$$
\tau_{j}:=\inf \left\{t \geq 0:\left(\left\|u_{k}^{n}\right\|_{Y_{k r}}^{q}+\left\|v_{j}\right\|_{Y_{t}}^{q}\right)^{\frac{1}{q}} \geq 2 n\right\} \wedge r
$$

for $j=1,2$. We follow the lines of the initial step where we replace $u_{j}$ by $v_{j}$ and $\theta_{n}\left(\left\|u_{j}\right\|_{Y_{s}}\right)$ by $\theta_{n}\left(\left(\left\|u_{k}^{n}\right\|_{Y_{k r}}^{q}+\left\|v_{j}\right\|_{Y_{s}}^{q}\right)^{\frac{1}{q}}\right)$ for $j=1,2$. We obtain

$$
\begin{aligned}
\left\|K_{d e t, k}^{n} v_{1}-K_{d e t, k}^{n} v_{2}\right\|_{E_{r}} \leq & \tau_{1}^{\delta}\left(\left\|v_{1}\right\|_{L^{q}\left(0, \tau_{1}, L^{\alpha+1}\right)}+\left\|v_{2}\right\|_{L^{q}\left(0, \tau_{1}, L^{\alpha+1}\right)}\right)^{\alpha-1}\left\|v_{1}-v_{2}\right\|_{E_{r}} \\
& +\frac{1}{n}\left\|v_{1}-v_{2}\right\|_{E_{r}} \tau_{2}^{\delta}\left\|v_{2}\right\|_{L^{q}\left(0, \tau_{2} ; L^{\alpha+1}\right)}^{\alpha}
\end{aligned}
$$

and by

$$
\left\|v_{j}\right\|_{L^{q}\left(0, \tau_{1}, L^{\alpha+1}\right)}=\left\|v_{j}\right\|_{Y_{\tau_{1}}} \leq\left(\left\|u_{k}^{n}\right\|_{Y_{k r}}^{q}+\left\|v_{j}\right\|_{Y_{\tau_{1}}}^{q}\right)^{\frac{1}{q}}=2 n, \quad j=1,2
$$


we conclude

$$
\begin{aligned}
\left\|K_{d e t, k}^{n} v_{1}-K_{d e t, k}^{n} v_{2}\right\|_{E_{r}} & \leq \tau_{1}^{\delta}(4 n)^{\alpha-1}\left\|v_{1}-v_{2}\right\|_{E_{r}}+\frac{1}{n}\left\|v_{1}-v_{2}\right\|_{E_{r}} \tau_{2}^{\delta}(2 n)^{\alpha} \\
& \leq r^{\delta}\left((4 n)^{\alpha-1}+\frac{1}{n}(2 n)^{\alpha}\right)\left\|v_{1}-v_{2}\right\|_{E_{r}} .
\end{aligned}
$$

Since the constant is the same as in the initial step, the definition of $r>0$ yields that $K_{k}^{n}$ is a strict contraction on $\mathbb{M}_{\mathbb{F}^{k r}}^{q}\left(\Omega, E_{r}\right)$. We call the unique fixed point $v_{k+1}^{n}$ and set

$$
u_{k+1}^{n}(t):= \begin{cases}u_{k}^{n}(t), & t \in[0, k r], \\ v_{k+1}^{n}(t-k r), & t \in[k r,(k+1) r] .\end{cases}
$$

Obviously, $u_{k+1}^{n}$ is a continuous $\mathbb{F}$-adapted process with $\left\|u_{k+1}^{n}\right\|_{L^{q}\left(\Omega, E_{(k+1) r}\right)}<\infty$ and therefore $u_{k+1}^{n} \in \mathbb{M}_{\mathbb{F}}^{q}\left(\Omega, E_{(k+1) r}\right)$. Let $t \in[k r,(k+1) r]$ and define $\tilde{t}:=t-k r$. Then, the definition of $K_{k}^{n}$ and the induction assumption yield

$$
\begin{aligned}
u_{k+1}^{n}(t)= & v_{k+1}^{n}(\tilde{t})=K_{k}^{n} v_{k+1}^{n}(\tilde{t})=U(\tilde{t}) u_{k}^{n}(k r)+K_{\text {det }, k}^{n} v_{k+1}^{n}(\tilde{t})+K_{\text {Strat }} v_{k+1}^{n}(\tilde{t})+K_{\text {stoch }, k} v_{k+1}^{n}(\tilde{t}) \\
= & U(t) u_{0}+\left[U(\tilde{t}) K_{d e t}^{n} u_{k}^{n}(k r)+K_{\text {det }, k}^{n} v_{k+1}^{n}(\tilde{t})\right]+\left[U(\tilde{t}) K_{\text {Strat }} u_{k}^{n}(k r)+K_{\text {Strat }} v_{k+1}^{n}(\tilde{t})\right] \\
& +\left[U(\tilde{t}) K_{\text {stoch }} u_{k}^{n}(r)+K_{\text {stoch }, k} v_{k+1}^{n}(\tilde{t})\right] .
\end{aligned}
$$

We compute

$$
\begin{aligned}
U(\tilde{t}) K_{d e t}^{n} u_{k}^{n}(k r)+K_{d e t, k}^{n} & v_{k+1}^{n}(\tilde{t})=-\mathrm{i} U(\tilde{t}) \int_{0}^{k r} U(k r-s)\left[\theta_{n}\left(\left\|u_{k}^{n}\right\|_{Y_{s}}\right)\left|u_{k}^{n}(s)\right|^{\alpha-1} u_{k}^{n}(s)\right] \mathrm{d} s \\
& -\mathrm{i} \int_{0}^{\tilde{t}} U(\tilde{t}-\tilde{s})\left[\theta_{n}\left(\left(\left\|u_{k}^{n}\right\|_{Y_{k r}}^{q}+\left\|v_{k+1}^{n}\right\|_{Y_{\tilde{s}}}^{q}\right)^{\frac{1}{q}}\right)\left|v_{k+1}^{n}(\tilde{s})\right|^{\alpha-1} v_{k+1}^{n}(\tilde{s})\right] \mathrm{d} \tilde{s} \\
= & -\mathrm{i} \int_{0}^{k r} U(t-s)\left[\theta_{n}\left(\left\|u_{k+1}^{n}\right\|_{Y_{s}}\right)\left|u_{k+1}^{n}(s)\right|^{\alpha-1} u_{k+1}^{n}(s)\right] \mathrm{d} s \\
& -\mathrm{i} \int_{0}^{\tilde{t}} U(\tilde{t}-\tilde{s})\left[\theta_{n}\left(\left\|u_{k+1}^{n}\right\|_{Y_{k r+\tilde{s}}}\right)\left|u_{k+1}^{n}(k r+\tilde{s})\right|^{\alpha-1} u_{k+1}^{n}(k r+\tilde{s})\right] \mathrm{d} \tilde{s} \\
= & -\mathrm{i} \int_{0}^{t} U(t-s)\left[\theta_{n}\left(\left\|u_{k+1}^{n}\right\|_{Y_{s}}\right)\left|u_{k+1}^{n}(s)\right|^{\alpha-1} u_{k+1}^{n}(s)\right] \mathrm{d} s \\
= & K_{d e t}^{n} u_{k+1}^{n}(t),
\end{aligned}
$$

where we used the substitution $s=k r+\tilde{s}$ in the second integral for the last step. Analogously,

$$
\begin{gathered}
U(\tilde{t}) K_{\text {Strat }} u_{k}^{n}(k r)+K_{\text {Strat }} v_{k+1}^{n}(\tilde{t})=K_{\text {Strat }} u_{k+1}^{n}(t), \\
U(\tilde{t}) K_{\text {stoch }} u_{k}^{n}(k r)+K_{\text {Stoch }, k} v_{k+1}^{n}(\tilde{t})=K_{\text {stoch }} u_{k+1}^{n}(t),
\end{gathered}
$$

where one uses (2.4) for the stochastic convolutions. Hence, we get

$$
u_{k+1}^{n}(t)=U(t) u_{0}+K_{\text {det }}^{n} u_{k+1}^{n}(t)+K_{\text {Strat }}^{n} u_{k+1}^{n}(t)+K_{\text {stoch }, k}^{n} u_{k+1}^{n}(t)=K^{n} u_{k+1}^{n}(t)
$$

for $t \in[k r,(k+1) r]$ and therefore, $u_{k+1}^{n}$ is a fixed point of $K^{n}$ in $\mathbb{M}_{\mathbb{F}}^{q}\left(\Omega, E_{(k+1) r}\right)$. Let $T>0$ and define $k:=\left\lfloor\frac{T}{r}+1\right\rfloor$. Then, $u^{n}:=u_{k}^{n}$ is the process from the assertion.

Step 3: Now, we turn our attention to uniqueness. Let $(\tilde{u}, \tau)$ be another local mild solution of (3.1). As in (3.7), we get

$$
\|u-\tilde{u}\|_{\mathbb{M}_{\mathbb{F}}^{q}\left(\Omega, E_{\tau \wedge r}\right)}=\left\|K^{n}(u)-K^{n}(\tilde{u})\right\|_{\mathbb{M}_{\mathbb{F}}^{q}\left(\Omega, E_{\tau \wedge r}\right)}
$$




$$
\begin{aligned}
& \leq C\left[\left(2^{\alpha}+4^{\alpha-1}\right) r^{\delta} n^{\alpha-1}+r^{\frac{1}{2}}+r\right]\|u-\tilde{u}\|_{\mathbb{M}_{\mathbb{F}}^{q}\left(\Omega, E_{\tau \wedge r}\right)} \\
& \leq \frac{1}{2}\|u-\tilde{u}\|_{\mathbb{M}_{\mathbb{F}}^{q}\left(\Omega, E_{\tau \wedge r}\right)},
\end{aligned}
$$

which leads to $u(t)=\tilde{u}(t)$ in $\mathbb{M}_{\mathbb{F}}^{q}\left(\Omega, E_{\tau \wedge r}\right)$, i.e. $u=\tilde{u}$ almost surely on $\{t \leq \tau \wedge r\}$. This can be iterated to see that $u(t)=\tilde{u}(t)$ almost surely on $\left\{t \leq \sigma_{k}\right\}$ with $\sigma_{k}:=\tau \wedge(k r)$ for $k \in \mathbb{N}$. The assertion follows from $\sigma_{k} \rightarrow \tau$ for $k \rightarrow \infty$.

In the following two Propositions, we use the results on the truncated equation (3.1) to derive existence and uniqueness for the original problem (1.1). The proofs are quite standard and in the literature, analogous arguments have been used in various contexts for extensions of wellposedness results from integrable to non-integrable initial values and from globally to locally Lipschitz nonlinearities, see for example [vNVW08], Theorem 7.1, [Brz97], Theorem 4.10, and [Sei93], Theorem 1.5.

Proposition 3.3. Let $\alpha \in\left(1,1+\frac{4}{d}\right)$ and $\left(u^{n}\right)_{n \in \mathbb{N}} \subset \mathbb{M}_{\mathbb{F}}^{q}\left(\Omega, E_{T}\right)$ be the sequence constructed in Proposition 3.2. For $n \in \mathbb{N}$, we define a stopping time $\tau_{n}$ by

$$
\tau_{n}:=\inf \left\{t \in[0, T]:\left\|u^{n}\right\|_{Y_{t}} \geq n\right\} \wedge T .
$$

Then, the following assertions hold:

a) We have $\tau_{n} \leq \tau_{k}$ almost surely for $n \leq k$ and $u^{n}(t)=u^{k}(t)$ almost surely on $\left\{t \leq \tau_{n}\right\}$.

b) The triple $\left(u,,\left(\tau_{n}\right)_{n \in \mathbb{N}}, \tau_{\infty}\right)$ with $u(t):=u^{n}(t)$ for $t \in\left[0, \tau_{n}\right]$ and $\tau_{\infty}:=\sup _{n \in \mathbb{N}} \tau_{n}$ is a local mild solution of (1.1).

Proof. ad a): Define

$$
\tau_{k, n}:=\inf \left\{t \in[0, T]:\left\|u^{k}\right\|_{Y_{t}} \geq n\right\} \wedge T \text {. }
$$

Then, we have $\tau_{k, n} \leq \tau_{n}$ and $\theta_{n}\left(\left\|u^{k}\right\|_{Y_{t}}\right)=1=\theta_{k}\left(\left\|u^{k}\right\|_{Y_{t}}\right)$ on $\left\{t \leq \tau_{k, n}\right\}$. Hence, $\left(u^{k}, \tau_{k, n}\right)$ is a solution of (3.1) and by the uniqueness part of Proposition 3.2, we obtain $u^{k}(t)=u^{n}(t)$ almost surely on $\left\{t \leq \tau_{k, n}\right\}$. But this leads to $\tau_{k, n}=\tau_{n}$ which implies the assertion.

$a d b$ ): By part a), $u$ is welldefined up to a null set, where we define $u:=0$ and $\tau_{\infty}=T$. The monotonicity of $\left(\tau_{n}\right)_{n \in \mathbb{N}}$ yields $\tau_{n} \rightarrow \tau$ almost surely. Moreover, $u \in \mathbb{M}_{\mathbb{F}}^{q}\left(\Omega, E_{\tau_{n}}\right)$ by Proposition 3.2 and therefore $u \in \mathbb{M}_{\mathbb{F}}^{q}\left(\Omega, E_{[0, \tau)}\right)$. From (3.6) and the identity

$$
\theta_{n}\left(\|u\|_{Y_{s}}\right)=\theta_{n}\left(\left\|u^{n}\right\|_{Y_{s}}\right)=1 \quad \text { a.s on }\left\{t \wedge \tau_{n}\right\}
$$

we finally obtain

$$
u(t)=U(t) u_{0}+\int_{0}^{t} U(t-s)\left[-\mathrm{i} \lambda|u(s)|^{\alpha-1} u(s)+\mu(u(s))\right] \mathrm{d} s-\mathrm{i} \int_{0}^{t} U(t-s) B u(s) \mathrm{d} W(s)
$$

almost surely on $\left\{t \leq \tau_{n}\right\}$ for all $n \in \mathbb{N}$.

Proposition 3.4. Let $\left(u_{1},\left(\sigma_{n}\right)_{n \in \mathbb{N}} \sigma\right),\left(u_{2},\left(\tau_{n}\right)_{n \in \mathbb{N}}, \tau\right)$ be local mild solutions to (1.1). Then,

$$
u_{1}(t)=u_{2}(t) \quad \text { a.s. on }\{t<\sigma \wedge \tau\}
$$

i.e. the solution of (1.1) is unique. 
Proof. We fix $k, n \in \mathbb{N}$ and define a stopping time by

$$
\nu_{k, n}:=\inf \left\{t \in[0, T]:\left\|u_{1}\right\|_{Y_{t}} \vee\left\|u_{2}\right\|_{Y_{t}} \geq n\right\} \wedge \sigma_{k} \wedge \tau_{k} .
$$

Hence, $\theta_{n}\left(\left\|u_{1}\right\|_{Y_{t}}\right)=\theta_{n}\left(\left\|u_{2}\right\|_{Y_{t}}\right)=1$ on $\left\{t \leq \nu_{k, n}\right\}$ and therefore $\left(u_{1}, \nu_{k, n}\right)$ and $\left(u_{2}, \nu_{k, n}\right)$ are local mild solutions of (3.1). By the uniqueness part of Proposition 3.2, we get

$$
u_{1}(t)=u_{2}(t) \quad \text { a.s. on }\left\{t \leq \nu_{k, n}\right\},
$$

which yields the assertion, since $\nu_{k, n} \rightarrow \sigma \wedge \tau$ almost surely for $n, k \rightarrow \infty$.

We continue with the critical case $\alpha=1+\frac{4}{d}$.

Proof of Theorem 1.1, b). Step 1. Let $\nu>0$ and $q:=2+\frac{4}{d}$. Then, $(q, q)$ is a Strichartz pair. For $r>0$, we define

$$
Y_{r}:=L^{q}\left(0, r ; L^{q}\left(\mathbb{R}^{d}\right)\right), \quad E_{r}:=C\left([0, r], L^{2}\left(\mathbb{R}^{d}\right)\right) \cap Y_{r}
$$

and as in the proof of Proposition 3.2, we set

$$
K_{1}^{\nu} u:=U(\cdot) u_{0}+K_{\text {det }}^{\nu} u+K_{\text {Strat }} u+K_{\text {stoch }} u
$$

with the convolution operators from (3.3), (3.4) and (3.5) and obtain the estimates

$$
\left\|K_{1}^{\nu} u\right\|_{L^{q}\left(\Omega, E_{r}\right)} \lesssim\left\|u_{0}\right\|_{L^{2}\left(\mathbb{R}^{d}\right)}+(2 \nu)^{\alpha}+\left(r+r^{\frac{1}{2}}\right)\|u\|_{L^{q}\left(\Omega, E_{r}\right)}
$$

and

$$
\left\|K_{1}^{\nu}\left(u_{1}\right)-K_{1}^{\nu}\left(u_{2}\right)\right\|_{L^{q}\left(\Omega, E_{r}\right)} \lesssim\left[\left(2^{\alpha}+4^{\alpha-1}\right) \nu^{\alpha-1}+r^{\frac{1}{2}}+r\right]\left\|u_{1}-u_{2}\right\|_{L^{q}\left(\Omega, E_{r}\right)} .
$$

for $u, u_{1}, u_{2} \in \mathbb{M}_{\mathbb{F}}^{q}\left(\Omega, E_{r}\right)$. Note that replacing the integer $n$ by $\nu>0$ in the cutoff function does not change the estimates at all. In fact, the only adaptation compared to the proof of Proposition 3.2 is due to $\delta:=1-\frac{d}{4}(1-\alpha)=0$ in the critical case. Choosing $\nu$ and $r$ small enough, we get a unique fixed point $u_{1} \in \mathbb{M}_{\mathbb{F}}^{q}\left(\Omega, E_{r}\right)$ of $K_{1}^{\nu}$.

By the definition of $\theta_{\nu}$, the fixed point $u_{1}$ from the first step is a solution of the original equation, as long as $\left\|u_{1}\right\|_{L^{q}\left(0, t ; L^{q}\right)} \leq \nu$. Hence, the pair $\left(u_{1}, \tau_{1}\right)$ with

$$
\tau_{1}:=\inf \left\{t \geq 0:\left\|u_{1}\right\|_{L^{q}\left(0, t ; L^{q}\right)} \geq \nu\right\} \wedge r
$$

is a local mild solution of 1.1 .

Step 2. Next, we define the operator

$$
K_{2}^{\nu} u:=U(\cdot) u_{1}\left(\tau_{1}\right)+K_{\text {det }}^{\nu} u+K_{\text {Strat }} u+K_{\text {stoch }, 2} u
$$

with

$$
K_{\text {stoch }, 2} u(t):=-\mathrm{i} J_{[0, r]}^{\tau_{1}} B u(t):=-\mathrm{i} \int_{0}^{t} U(t-s) B u(s) \mathrm{d} W^{\tau_{1}}(s)
$$

and analogously as above, we derive the estimates

$$
\left\|K_{2}^{\nu} u\right\|_{L^{q}\left(\Omega, E_{r}\right)} \lesssim\left\|u_{1}\left(\tau_{1}\right)\right\|_{L^{q}\left(\Omega, L^{2}\left(\mathbb{R}^{d}\right)\right)}+(2 \nu)^{\alpha}+\left(r+r^{\frac{1}{2}}\right)\|u\|_{L^{q}\left(\Omega, E_{r}\right)}
$$

and

$$
\left\|K_{2}^{\nu}\left(u_{1}\right)-K_{2}^{\nu}\left(u_{2}\right)\right\|_{L^{q}\left(\Omega, E_{r}\right)} \lesssim\left[\left(2^{\alpha}+4^{\alpha-1}\right) \nu^{\alpha-1}+r^{\frac{1}{2}}+r\right]\left\|u_{1}-u_{2}\right\|_{L^{q}\left(\Omega, E_{r}\right)} .
$$

for $u, u_{1}, u_{2} \in \mathbb{M}_{\mathbb{F}^{\tau_{1}}}^{q}\left(\Omega, E_{r}\right)$. We get a unique fixed point $\tilde{u}_{2} \in \mathbb{M}_{\mathbb{F}^{\tau_{1}}}^{q}\left(\Omega, E_{r}\right)$ of $K_{2}^{\nu}$ and define

$$
\tilde{\tau}_{2}:=\inf \left\{t \geq 0:\left\|u_{2}\right\|_{L^{q}\left(0, t ; L^{q}\right)} \geq \nu\right\} \wedge r
$$


and $\tau_{2}:=\tau_{1}+\tilde{\tau}_{2}$. Analogously to the proof of Proposition 3.2, one can show using (2.4), that the pair $\left(u_{2}, \tau_{2}\right)$ with

$$
u_{2}(t):= \begin{cases}u_{1}(t), & t \in\left[0, \tau_{1}\right], \\ \tilde{u}_{2}\left(t-\tau_{1}\right), & t \in\left[\tau_{1}, \tau_{2}\right] .\end{cases}
$$

defines a local mild solution of (1.1). Iterating this procedure yields a sequence $\left(u_{n}, \tau_{n}\right)_{n \in \mathbb{N}}$ and with $\tau_{\infty}:=\sup _{n \in \mathbb{N}} \tau_{n}$ and

$$
u(t):=\mathbf{1}_{\{t=0\}} u_{0}+\sum_{n=1}^{\infty} u_{n}(t) \mathbf{1}_{{\left(\tau_{n-1}, \tau_{n}\right]}}(t) \quad \text { on }\left\{t \leq \tau_{\infty}\right\},
$$

the triple $\left(u,\left(\tau_{n}\right)_{n \in \mathbb{N}}, \tau_{\infty}\right)$ is a local mild solution in the sense of Definition 2.4 .

Step 3. To show uniqueness, we take two local mild solutions $\left(u_{1},\left(\sigma_{1, n}\right)_{n \in \mathbb{N}} \sigma_{1}\right)$ and $\left(u_{2},\left(\sigma_{2, n}\right)_{n \in \mathbb{N}}, \sigma_{2}\right)$ and define a sequence of stopping times by

$$
\mu_{1}:=\inf \left\{t \in\left[0, \sigma_{1}\right):\left\|u_{1}\right\|_{Y_{[0, t]}} \geq \nu\right\} \wedge \inf \left\{t \in\left[0, \sigma_{2}\right):\left\|u_{2}\right\|_{Y_{[0, t]}} \geq \nu\right\} \wedge \sigma_{1} \wedge \sigma_{2}
$$

and

$$
\mu_{n+1}:=\inf \left\{t \in\left[\mu_{n}, \sigma_{1}\right):\left\|u_{1}\right\|_{Y_{\left[\mu_{n}, t\right]}} \geq \nu\right\} \wedge \inf \left\{t \in\left[\mu_{n}, \sigma_{2}\right):\left\|u_{2}\right\|_{Y_{\left[\mu_{n}, t\right]}} \geq \nu\right\} \wedge \sigma_{1} \wedge \sigma_{2} .
$$

The uniqueness from the first step and $\theta_{\nu}\left(\left\|u_{1}\right\|_{Y_{t}}\right)=1=\theta_{\nu}\left(\left\|u_{2}\right\|_{Y_{t}}\right)$ almost surely on $\left\{t \leq \mu_{1}\right\}$ yield $u_{1}(t)=u_{2}(t)$ almost surely on $\left\{t<\sigma_{1} \wedge \sigma_{2}\right\} \cap\left\{t \leq \mu_{1}\right\}$. By an iteration procedure as above, this can be extended to $\left\{t<\sigma_{1} \wedge \sigma_{2}\right\} \cap\left\{t \leq \mu_{n}\right\}$ for all $n \in \mathbb{N}$.

In order to show, that $\mu_{n} \rightarrow \sigma_{1} \wedge \sigma_{2}$ as $n \rightarrow \infty$, it is sufficient that for all $m \in \mathbb{N}$ and almost all $\omega \in \Omega$ there is $n=n(\omega)$ with $\mu_{n(\omega)}(\omega) \geq \sigma_{1, m}(\omega) \wedge \sigma_{2, m}(\omega)$. Assume the opposite, i.e. there is $m \in \mathbb{N}$ with

$$
\mathbb{P}\left(\mu_{n}<\sigma_{1, m} \wedge \sigma_{2, m} \quad \forall n \in \mathbb{N}\right)>0 .
$$

By the definition of $\mu_{n+1}$, we get on each interval $\left[\mu_{n}, \mu_{n+1}\right]$ either $\left\|u_{1}\right\|_{Y_{\left[\mu_{n}, \mu_{n+1}\right]}} \geq \nu$ or $\left\|u_{2}\right\|_{Y_{\left[\mu_{n}, \mu_{n+1}\right]}} \geq \nu$ with positive probability. Without loss of generality, we assume that there is a subsequence $\left(\mu_{n_{k}}\right)_{k \in \mathbb{N}}$ with

$$
\mathbb{P}\left(\left\|u_{1}\right\|_{Y_{\left[\mu_{k}, \mu_{n_{k}+1}\right]}} \geq \nu \quad \forall k \in \mathbb{N}\right)>0
$$

and therefore

$$
\left\|u_{1}\right\|_{Y_{\sigma_{1, m}}} \geq\left(\sum_{k=1}^{\infty}\left\|u_{1}\right\|_{Y_{\left[\mu_{n_{k}}, \mu_{n_{k}}+1\right]}}^{q}\right)^{\frac{1}{q}}=\infty
$$

which contradicts $\left\|u_{1}\right\|_{Y_{\sigma_{1, m}}}<\infty$ almost surely by $u_{1} \in \mathbb{M}_{\mathbb{F}}^{q}\left(\Omega, E_{\left[0, \sigma_{1}\right)}\right)$.

We close this section with a remark on possible slight generalizations of Theorem 1.1 and a comment on the transfer of our method to the energy space $H^{1}\left(\mathbb{R}^{d}\right)$.

Remark 3.5. In the proof of local wellposedness, we did not use the special structure of the term

$$
\mu:=-\frac{1}{2} \sum_{m=1}^{\infty} B_{m}^{*} B_{m}
$$


In fact, we only used $\mu \in \mathcal{L}\left(L^{2}\left(\mathbb{R}^{d}\right)\right)$. But since (3.9) is motivated by the Stratonovich noise and will be important for the global existence in the following section, we decided to start with it from the beginning.

A generalization of the result from Theorem 1.1 from determistic initial values $u_{0} \in L^{2}\left(\mathbb{R}^{d}\right)$ to $u_{0} \in L^{q}\left(\Omega, \mathcal{F}_{0} ; L^{2}\left(\mathbb{R}^{d}\right)\right)$ is straightforward. By the standard localization technique (see e.g. [vNVW08]), a further generalization to $\mathcal{F}_{0}$-measurable $u_{0}: \Omega \rightarrow L^{2}\left(\mathbb{R}^{d}\right)$ can be done if one relaxes the condition $u \in \mathbb{M}_{\mathbb{F}}^{q}\left(\Omega, E_{[0, \tau)}\right)$ from definition 2.4 to $u \in \mathbb{M}_{\mathbb{F}}^{0}\left(\Omega, E_{[0, \tau)}\right)$, i.e. $u$ is a continuous $\mathbb{F}$-adapted process in $L^{2}\left(\mathbb{R}^{d}\right)$ with

$$
\sup _{t \in[0, \tau]}\|u(t)\|_{L^{2}}+\left(\int_{0}^{\tau}\|u(t)\|_{L^{\alpha+1}}^{q} \mathrm{~d} t\right)^{\frac{1}{q}}<\infty \quad \text { a.s. }
$$

For the sake of simplicity, we decided to restrict to deterministic initial values.

Remark 3.6. Barbu, Röckner and Zhang, [BRZ16], and de Bouard and Debussche, [dBD03], also applied their strategy to construct solutions also in $H^{1}\left(\mathbb{R}^{d}\right)$. In contrast to the $L^{2}$-case, the pathwise approach has a true advantage here, since it allows to adapt the deterministic fixed point argument in a ball of $L^{\infty} H^{1} \cap L^{q} W^{1, \alpha+1}$ equipped with the metric from $L^{\infty} L^{2} \cap L^{q} L^{\alpha+1}$. Therefore, [BRZ16] gets wellposedness for all $H^{1}$-subcritical exponents $\alpha \in\left(1,1+\frac{4}{(d-2)_{+}}\right)$.

Of course, it is also possible to deal with the $H^{1}$-problem with the method from the present paper, since the deterministic and stochastic Strichartz estimates are also true in $H^{1}\left(\mathbb{R}^{d}\right)$. In this way, one can weaken the assumptions on the noise from [BRZ16] significantly to

$$
\sum_{m=1}^{\infty}\left\|e_{m}\right\|_{W^{1, \infty}}^{2}<\infty
$$

But unfortunately, the use of the truncated equation cannot be combined with two different norms for the invariance and the contraction estimate. On the other hand, proving the contraction in the full norm of $L^{\infty} H^{1} \cap L^{q} W^{1, \alpha+1}$ requires a restriction to $\alpha \in\left(2,1+\frac{4}{(d-2)_{+}}\right)$.

\section{GLOBAL WELlPOSEDNESS IN THE SUBCRITICAL CASE}

Having established local results, the next goal is to get global wellposedness. The first ingredient is an estimate of the $L^{2}$-norm of solutions.

Proposition 4.1. Let $\alpha \in\left(1,1+\frac{4}{d}\right), n \in \mathbb{N}$ and $u_{n}$ be the global mild solution of (3.1) from Proposition 3.2. Then, we have

$$
\left\|u_{n}(t)\right\|_{L^{2}\left(\mathbb{R}^{d}\right)}^{2}=\left\|u_{0}\right\|_{L^{2}\left(\mathbb{R}^{d}\right)}^{2}-2 \int_{0}^{t} \operatorname{Re}\left(u_{n}(s), \mathrm{i} B u_{n}(s) \mathrm{d} W(s)\right)_{L^{2}}, \quad t \in[0, T] .
$$

almost surely. Moreover, for all $p \in[1, \infty)$, there is a constant $D_{p}=D_{p}\left(T,\left\|u_{0}\right\|_{L^{2}}\right)>0$ independent of $n$ with

$$
\mathbb{E}\left[\sup _{t \in[0, T]}\left\|u_{n}(t)\right\|_{L^{2}}^{p}\right] \leq D_{p}
$$

Note that the estimate (4.1) for $p=2$ previously occured in [BRZ14b] and in the special case of Stratonovich noise with selfadjoint operators $B_{m}, m \in \mathbb{N}$, (4.1) simplifies to $\left\|u_{n}(t)\right\|_{L^{2}}=\left\|u_{0}\right\|_{L^{2}}$ almost surely for all $t \in[0, T]$. This generalizes the $L^{2}$-conservation of the NLS, see [LP14], equation (6.2), to the stochastic setting. 
Proof. Step 1. It is well known that the mild equation is equivalent to

$$
u_{n}(t)=u_{0}+\int_{0}^{t}\left[\mathrm{i} \Delta u_{n}(s)-\mathrm{i} \lambda \theta_{n}\left(\left\|u_{n}\right\|_{Y_{s}}\right)\left|u_{n}(s)\right|^{\alpha-1} u_{n}(s)+\mu\left(u_{n}(s)\right)\right] \mathrm{d} s-\mathrm{i} \int_{0}^{t} B u_{n}(s) \mathrm{d} W(s)
$$

almost surely as an equation in $H^{-2}\left(\mathbb{R}^{d}\right)$. We formally apply Ito's formula to the Itô process from (4.2) and the function $\mathcal{M}: L^{2}\left(\mathbb{R}^{d}\right) \rightarrow \mathbb{R}$ defined by $\mathcal{M}(v):=\|v\|_{L^{2}}^{2}$, which is twice continuously Fréchet-differentiable with

$$
\mathcal{M}^{\prime}[v] h_{1}=2 \operatorname{Re}\left(v, h_{1}\right)_{L^{2}}, \quad \mathcal{M}^{\prime \prime}[v]\left[h_{1}, h_{2}\right]=2 \operatorname{Re}\left(h_{1}, h_{2}\right)_{L^{2}}
$$

for $v, h_{1}, h_{2} \in L^{2}\left(\mathbb{R}^{d}\right)$. This yields

$$
\begin{aligned}
\left\|u_{n}(t)\right\|_{L^{2}}^{2}= & \left\|u_{0}\right\|_{L^{2}}^{2}+2 \int_{0}^{t} \operatorname{Re}\left(u_{n}(s), \mathrm{i} \Delta u_{n}(s)-\mathrm{i} \theta_{n}\left(\left\|u_{n}\right\|_{Y_{s}}\right) \lambda\left|u_{n}(s)\right|^{\alpha-1} u_{n}(s)+\mu\left(u_{n}(s)\right)\right)_{L^{2}} \mathrm{~d} s \\
& -2 \int_{0}^{t} \operatorname{Re}\left(u_{n}(s), \mathrm{i} B u_{n}(s) \mathrm{d} W(s)\right)_{L^{2}}+\sum_{m=1}^{\infty} \int_{0}^{t}\left\|B_{m} u_{n}(s)\right\|_{L^{2}}^{2} \mathrm{~d} s
\end{aligned}
$$

almost surely in $[0, T]$ and using the formal identities

$$
\left(u_{n}, \mathrm{i} \Delta u_{n}\right)_{L^{2}}=0, \quad \operatorname{Re}\left(u_{n}, \mathrm{i} \theta_{n}\left(\left\|u_{n}\right\|_{Y .}\right)\left|u_{n}\right|^{\alpha-1} u_{n}\right)_{L^{2}}=0,
$$

and

$$
2 \operatorname{Re}\left(u_{n}, \mu\left(u_{n}\right)\right)_{L^{2}}=-\sum_{m=1}^{\infty}\left(u_{n}, B_{m}^{*} B_{m} u_{n}\right)_{L^{2}}=-\sum_{m=1}^{\infty}\left\|B_{m} u_{n}\right\|_{L^{2}}^{2},
$$

we obtain the first assertion.

The calculation from above can be made rigorous by a regularization procedure via Yosida approximations $R_{\nu}:=\lambda(\nu-\Delta)^{-1}$ for $\nu>0$ and a limit process $\nu \rightarrow \infty$ using the properties $R_{\nu} \in \mathcal{L}\left(H^{s}\left(\mathbb{R}^{d}\right), H^{s+2}\left(\mathbb{R}^{d}\right)\right)$ for $s \in \mathbb{R}$ and

$$
\begin{gathered}
R_{\nu} f \rightarrow f \text { in } E, \quad \nu \rightarrow \infty, \quad f \in E \\
\left\|R_{\nu}\right\|_{\mathcal{L}(E)} \leq 1
\end{gathered}
$$

for $E=H^{s}\left(\mathbb{R}^{d}\right), s \in \mathbb{R}$, and $E=L^{p}\left(\mathbb{R}^{d}\right), 1<p<\infty$.

Step 2. The estimate (4.1) follows from Step 1 by an application of the Burkholder-GundyDavis inequality to the stochastic integral and a Gronwall type argument. For more details, we refer the reader to [BRZ14b], Lemma 4.3. Note that they prove (4.1) only for $p=2$, but one can generalize this by using higher moments in the Burkholder-Gundy-Davis inequality.

In order to get global existence, we adapt an argument by de Bouard and Debussche, see [dBD99], Proposition 4.1, to our setting.

Proposition 4.2. Let $\alpha \in\left(1,1+\frac{4}{d}\right)$ and $\left(u^{n}\right)_{n \in \mathbb{N}}$ be the sequence of solutions to (3.1) from Proposition 3.2 and

$$
\tau_{n}:=\inf \left\{t \geq 0:\left\|u^{n}\right\|_{Y_{t}} \geq n\right\} \wedge T, \quad n \in \mathbb{N} .
$$


Then, we have

$$
\mathbb{P}\left(\bigcup_{n \in \mathbb{N}}\left\{\tau_{n}=T\right\}\right)=1 .
$$

In particular, the pair $\left(u, \tau_{\infty}\right)$ from Proposition 3.3 is a global mild solution of (1.1).

Proof. First, we recall some notions from the previous section. The exponent $q \in(2, \infty)$ is fixed according to

$$
\frac{2}{q}+\frac{d}{\alpha+1}=\frac{d}{2}
$$

and we set

$$
Y_{r}:=L^{q}\left(0, r ; L^{\alpha+1}\left(\mathbb{R}^{d}\right)\right), \quad E_{r}:=Y_{r} \cap C\left([0, r], L^{2}\left(\mathbb{R}^{d}\right)\right), \quad r>0 .
$$

Step 1. Let $T>0$. As a first step, we want to prove, that there is a constant $C=C\left(\left\|u_{0}\right\|_{L^{2}}, T\right)>$ 0 such that

$$
\sup _{n \in \mathbb{N}} \mathbb{E}\left\|u_{n}\right\|_{Y_{T}} \leq C .
$$

We fix $n \in \mathbb{N}$ and recall that $u_{n}$ has the representation

$$
u_{n}=U(\cdot) u_{0}+K_{\text {det }} u_{n}+K_{\text {Strat }} u_{n}+K_{\text {Stoch }} u_{n} \quad \text { in } L_{\mathbb{F}}^{q}\left(\Omega, C\left([0, T], L^{2}\left(\mathbb{R}^{d}\right)\right) \cap L^{q}\left(0, T ; L^{\alpha+1}\left(\mathbb{R}^{d}\right)\right)\right. \text {. }
$$

We fix a path $\omega \in \Omega$ and $\sigma_{n}(\omega) \in[0, T]$ to be chosen later. Let $\delta:=1-\frac{d}{4}(1-\alpha)$. Then, we apply the deterministic Strichartz inequalities from Proposition 2.2 to estimate $K_{\text {det }}$ and $K_{\text {Strat }}$ (compare the proof of Proposition 3.2) and obtain

$$
\begin{aligned}
\left\|u_{n}\right\|_{Y_{\sigma_{n}}} & \leq C\left\|u_{0}\right\|_{L^{2}}+C \sigma_{n}^{\delta}\left\|u_{n}\right\|_{Y_{\sigma_{n}}}^{\alpha}+C\left\|u_{n}\right\|_{L^{1}\left(0, \sigma_{n}, L^{2}\right)} \sum_{m=1}^{\infty}\left\|B_{m}\right\|_{\mathcal{L}\left(L^{2}\right)}^{2}+\left\|K_{S t o c h} u_{n}\right\|_{Y_{\sigma_{n}}} \\
& \leq K_{n}+C \sigma_{n}^{\delta}\left\|u_{n}\right\|_{Y_{\sigma_{n}}}^{\alpha}
\end{aligned}
$$

where $K_{n}$ is defined by

$$
K_{n}:=C\left\|u_{n}\right\|_{L^{\infty}\left(0, T ; L^{2}\right)}\left(1+T \sum_{m=1}^{\infty}\left\|B_{m}\right\|_{\mathcal{L}\left(L^{2}\right)}^{2}\right)+\left\|K_{\text {Stoch }} u_{n}\right\|_{Y_{T}} .
$$

We conclude

$$
\frac{\left\|u_{n}\right\|_{Y_{\sigma_{n}}}}{K_{n}} \leq 1+C \sigma_{n}^{\delta} K_{n}^{\alpha-1}\left(\frac{\left\|u_{n}\right\|_{Y_{\sigma_{n}}}}{K_{n}}\right)^{\alpha}
$$

Now, the following fact

$$
\forall x \geq 0 \exists c_{1} \leq 2, c_{2}>c_{1}: \quad x \leq 1+\frac{x^{\alpha}}{2^{\alpha+1}} \quad \Rightarrow \quad x \leq c_{1} \quad \text { or } \quad x \geq c_{2} .
$$

from elementary calculus yields

$$
\left\|u_{n}\right\|_{Y_{\sigma_{n}}} \leq 2 c_{1} \leq 2 K_{n}
$$

if we choose $\sigma_{n}$ according to $C \sigma_{n}^{\delta} K_{n}^{\alpha-1} \leq \frac{1}{2^{\alpha+1}}$, which is fulfilled by

$$
\sigma_{n}=C^{-\frac{1}{\delta}}\left(2^{\alpha+1} K_{n}^{\alpha-1}\right)^{-\frac{1}{\delta}} \wedge T \text {. }
$$


Note that the second alternative in (4.7) can be excluded because of $\left\|u_{n}\right\|_{Y_{0}}=0$ and the continuity of the map $t \mapsto\left\|u_{n}\right\|_{Y_{t}}$. Next, we decompose $\Omega=\Omega_{1} \cup \Omega_{2}$ with

$$
\Omega_{1}:=\left\{C^{-\frac{1}{\delta}}\left(2^{\alpha+1} K_{n}^{\alpha-1}\right)^{-\frac{1}{\delta}}<T\right\}, \quad \Omega_{2}:=\left\{C^{-\frac{1}{\delta}}\left(2^{\alpha+1} K_{n}^{\alpha-1}\right)^{-\frac{1}{\delta}} \geq T\right\} .
$$

Fix $\omega \in \Omega_{1}$ and define $N:=\left\lfloor\frac{T}{\sigma_{n}}\right\rfloor$. Using the abbreviation

$$
Y_{j}:=L^{q}\left(j \sigma_{n},(j+1) \sigma_{n} ; L^{\alpha+1}\left(\mathbb{R}^{d}\right)\right), \quad j=0, \ldots, N,
$$

we get

$$
\begin{aligned}
\left\|u_{n}\right\|_{Y_{j}} & \leq C\left\|u_{n}\left(j \sigma_{n}\right)\right\|_{L^{2}}+C \sigma_{n}^{\delta}\left\|u_{n}\right\|_{Y_{j}}^{\alpha}+C T\left\|u_{n}\right\|_{L^{\infty}\left(j \sigma_{n},(j+1) \sigma_{n} ; L^{2}\right)} \sum_{m=1}^{\infty}\left\|B_{m}\right\|_{\mathcal{L}\left(L^{2}\right)}^{2}+\left\|K_{S t o c h} u_{n}\right\|_{Y_{j}} \\
& \leq K_{n}+C \sigma_{n}^{\delta}\left\|u_{n}\right\|_{Y_{j}}^{\alpha}
\end{aligned}
$$

for all $j=0, \ldots, N$ by analogous estimates as in (4.6) and thus again $\left\|u_{n}\right\|_{Y_{j}} \leq 2 K_{n}$. We conclude

$$
\left\|u_{n}\right\|_{Y_{T}} \leq \sum_{j=0}^{N}\left\|u_{n}\right\|_{Y_{j}} \leq 2(N+1) K_{n} \leq 2\left(\frac{T}{\sigma_{n}}+1\right) K_{n} \lesssim 2 K_{n}+2 T K_{n}^{\frac{\alpha-1}{\delta}+1} .
$$

Since we have $\left\|u_{n}\right\|_{Y_{T}} \leq 2 K_{n}$ on $\Omega_{2}$, the estimate (4.8) holds almost surely. Then, we integrate over $\Omega$ to obtain

$$
\left\|u_{n}\right\|_{L^{1}\left(\Omega, Y_{T}\right)} \lesssim 2 \mathbb{E}\left[K_{n}\right]+2 T \mathbb{E}\left[K_{n}^{\frac{\alpha-1}{\delta}+1}\right]
$$

For the first term, we get

$$
\begin{aligned}
\mathbb{E}\left[K_{n}\right] & \leq C \mathbb{E}\left\|u_{n}\right\|_{L^{\infty}\left(0, T, L^{2}\right)}\left(1+T \sum_{m=1}^{\infty}\left\|B_{m}\right\|_{\mathcal{L}\left(L^{2}\right)}^{2}\right)+\left(\mathbb{E}\left\|K_{S t o c h} u_{n}\right\|_{Y_{T}}^{2}\right)^{\frac{1}{2}} \\
& \leq C D_{2}\left(1+T \sum_{m=1}^{\infty}\left\|B_{m}\right\|_{\mathcal{L}\left(L^{2}\right)}^{2}\right)+T^{\frac{1}{2}} D_{2}\left(\sum_{m=1}^{\infty}\left\|B_{m}\right\|_{\mathcal{L}\left(L^{2}\right)}^{2}\right)^{\frac{1}{2}}
\end{aligned}
$$

whereas for the second one, we write

$$
\mathbb{E}\left[K_{n}^{\frac{\alpha-1}{\delta}+1}\right] \lesssim \mathbb{E}\left[\left\|u_{n}\right\|_{L^{\infty}\left(0, T ; L^{2}\right)}^{\frac{\alpha-1}{\delta}+1}\right]+\mathbb{E}\left[\left\|K_{S t o c h} u_{n}\right\|_{Y_{T}}^{\frac{\alpha-1}{\delta}+1}\right]
$$

and apply Proposition 2.3 and Hölder's inequality in time as well as Proposition 4.1 for

$$
\mathbb{E}\left[\left\|K_{S t o c h} u_{n}\right\|_{Y_{T}}^{\frac{\alpha-1}{\delta}+1}\right] \lesssim \mathbb{E}\left[\left\|u_{n}\right\|_{L^{2}\left(0, T ; L^{2}\right)}^{\frac{\alpha-1}{\delta}+1}\right] \leq T^{\frac{\alpha-1}{2 \delta}+\frac{1}{2}} \mathbb{E}\left[\left\|u_{n}\right\|_{L^{\infty}\left(0, T ; L^{2}\right)}^{\frac{\alpha-1}{\delta}+1}\right] \leq T^{\frac{\alpha-1}{2 \delta}+\frac{1}{2}} D_{\frac{\alpha-1}{\delta}+1} .
$$

Hence, we have proved

$$
\sup _{n \in \mathbb{N}} \mathbb{E}\left\|u_{n}\right\|_{Y_{T}} \leq C\left(\left\|u_{0}\right\|_{L^{2}}, T, \alpha\right)
$$

Step 2. Recall $\tau_{\infty}:=\sup _{n \in \mathbb{N}} \tau_{n}$. By the definition of $\tau_{n}$ and (4.10), we obtain

$$
\mathbb{P}\left(\tau_{n}=T\right)=\mathbb{P}\left(\left\|u_{n}\right\|_{Y_{\sigma_{n}}} \leq n\right) \geq 1-\frac{C}{n}
$$

and using the continuity of the measure, we conclude

$$
\mathbb{P}\left(\tau_{\infty}=T\right) \geq \mathbb{P}\left(\bigcup_{n \in \mathbb{N}}\left\{\tau_{n}=T\right\}\right)=1
$$


We remark that the proof of the last Proposition heavily relies on $\delta>0$. Therefore, this strategy cannot be applied to the critical case $\alpha=1+\frac{4}{d}$, where we have $\delta=0$. But global existence cannot be expected in this case, anyway, since there are blow-up examples in the deterministic setting for the focusing nonlinearity, see [Mer93].

Acknowledgement: The author gratefully acknowledges financial support by the Deutsche Forschungsgemeinschaft (DFG) through CRC 1173.

\section{REFERENCES}

[BCI ${ }^{+}$94] O. Bang, P. L. Christiansen, F. If, K. Ø. Rasmussen, and Y. B. Gaididei. Temperature effects in a nonlinear model of monolayer Scheibe aggregates. Phys. Rev. E, 49:4627-4636, May 1994.

[BGT04] N. Burq, P. Gérard, and N. Tzvetkov. Strichartz inequalities and the nonlinear Schrödinger equation on compact manifolds. American Journal of Mathematics, 126 (3):569-605, 2004.

[BM13] Z. Brzeźniak and A. Millet. On the stochastic Strichartz estimates and the stochastic nonlinear Schrödinger equation on a compact riemannian manifold. Potential Analysis, 41(2):269-315, 2013.

[Brz97] Z. Brzeźniak. On stochastic convolution in Banach spaces and applications. Stochastics and Stochastic Reports, 61(3-4):245-295, 1997.

[BRZ14a] V. Barbu, M. Röckner, and D. Zhang. Stochastic nonlinear Schrödinger equations: no blow-up in the non-conservative case. preprint, 2014. arXiv: 1409.4007.

[BRZ14b] V. Barbu, M. Röckner, and D. Zhang. Stochastic nonlinear Schrödinger equations with linear multiplicative noise: Rescaling approach. Journal of Nonlinear Science, 24(3):383-409, 2014.

[BRZ16] V. Barbu, M. Röckner, and D. Zhang. Stochastic nonlinear Schrödinger equations. Nonlinear Analysis: Theory, Methods E Applications, 136:168-194, 2016.

[Caz03] T. Cazenave. Semilinear Schrödinger equations. Courant lecture notes in mathematics ; 10. Courant Institute of Mathematical Sciences, New York, NY, 2003.

[dBD99] A. de Bouard and A. Debussche. A stochastic nonlinear Schrödinger equation with multiplicative noise. Communications in Mathematical Physics, 205(1):161$181,1999$.

[dBD02] A. de Bouard and A Debussche. On the effect of a noise on the solutions of the focusing supercritical nonlinear Schrödinger equation. Probability theory and related fields, 123(1):76-96, 2002.

[dBD03] A. de Bouard and A. Debussche. The stochastic nonlinear Schrödinger equation in h 1. Stochastic Analysis and Applications, 21(1):97-126, 2003.

$\left[\mathrm{dBD}^{+} 05\right]$ A. de Bouard, A. Debussche, et al. Blow-up for the stochastic nonlinear Schrödinger equation with multiplicative noise. The Annals of Probability, 33(3):1078-1110, 2005.

[DBD06] A. De Bouard and A. Debussche. Weak and strong order of convergence of a semidiscrete scheme for the stochastic nonlinear Schrödinger equation. Applied Mathematics and Optimization, 54(3):369-399, 2006. 
[DDM02] A. Debussche and L. Di Menza. Numerical simulation of focusing stochastic nonlinear Schrödinger equations. Physica D: Nonlinear Phenomena, 162(3):131154, 2002.

[Doi96] S.-I. Doi. Remarks on the Cauchy problem for Schrödinger-type equations. Communications in Partial Differential Equations, 21(1-2):163-178, 1996.

[DPZ14] G. Da Prato and P.J. Zabczyk. Stochastic Equations in Infinite Dimensions. Encyclopedia of Mathematics and Its Applications. Cambridge University Press, 2014.

[LP14] F. Linares and G. Ponce. Introduction to nonlinear dispersive equations. Springer, 2014.

[Mer93] F. Merle. Determination of blow-up solutions with minimal mass for nonlinear Schrödinger equations with critical power. Duke Math. J., 69(2):427-454, 021993.

[MMT08] J. Marzuola, J. Metcalfe, and D. Tataru. Strichartz estimates and local smoothing estimates for asymptotically flat Schrödinger equations. Journal of Functional Analysis, 255(6):14971553, September 2008.

[Sei93] J. Seidler. Da Prato-Zabczyk's maximal inequality revisited. i. Mathematica Bohemica, 118(1):67-106, 1993.

[vNVW07] J.M.A.M. van Neerven, M. C. Veraar, and L. Weis. Stochastic integration in UMD Banach spaces. Ann. Probab., 35(4):1438-1478, 072007.

[vNVW08] J.M.A.M. van Neerven, M.C. Veraar, and L. Weis. Stochastic evolution equations in UMD Banach spaces. Journal of Functional Analysis, 255(4):940 - 993, 2008.

F. Hornung, Institute for Analysis, Karlsruhe Institute for Technology (KIT), 76128 Karlsruhe, Germany

E-mail address: fabian.hornung@kit.edu 